\title{
Criminologie
}

\section{Que deviennent les adolescentes judiciarisées près de dix ans après leur sortie du Centre jeunesse?}

\section{Nadine Lanctôt}

Volume 38, numéro 1, printemps 2005

Filles et déviance : perspectives développementales

URI : https://id.erudit.org/iderudit/011488ar

DOI : https://doi.org/10.7202/011488ar

Aller au sommaire du numéro

\section{Éditeur(s)}

Les Presses de l'Université de Montréal

ISSN

0316-0041 (imprimé)

1492-1367 (numérique)

Découvrir la revue

Citer cet article

Lanctôt, N. (2005). Que deviennent les adolescentes judiciarisées près de dix ans après leur sortie du Centre jeunesse ? Criminologie, 38(1), 139-162.

https://doi.org/10.7202/011488ar
Résumé de l'article

Cette étude vise trois objectifs : 1) établir la prévalence des conduites déviantes manifestées au cours de l'adolescence et au début de l'âge adulte par les jeunes femmes ayant été prises en charge par la justice au cours de l'adolescence 2) déterminer les trajectoires de l'activité déviante de ces jeunes femmes, de l'adolescence au début de l'âge adulte 3) évaluer la proportion des adolescentes judiciarisées qui sont aux prises avec des difficultés personnelles et sociales au début de l'âge adulte. L'échantillon est composé de 97 jeunes femmes qui ont fait l'objet d'une ordonnance de la Chambre de la jeunesse de Montréal en 1992-1993. Les données proviennent de questionnaires autorévélés qui ont été complétés par les participantes à trois reprises : à 15 ans, à 17 ans et à 23 ans. Les résultats révèlent que, malgré le fait que certaines adolescentes aient été fortement impliquées dans la déviance à la mi-adolescence, aucune trajectoire ne se démarque par une participation persistante à des actes violents ou à des délits contre la propriété. D’autres difficultés compromettent toutefois le bien-être de ces jeunes femmes. Les adolescentes judiciarisées sont particulièrement à risque de se retrouver, au début de l'âge adulte, dans des situations où la monoparentalité, la pauvreté, la faible scolarisation, la violence conjugale et la détresse se juxtaposent. 


\title{
Que deviennent les adolescentes judiciarisées près de dix ans après leur sortie du Centre jeunesse ? $^{1}$
}

\author{
Nadine Lanctôt ${ }^{2}$ \\ Professeure \\ École de criminologie \\ Université de Montréal \\ nadine.lanctot@umontreal.ca
}

RÉSUMÉ - Cette étude vise trois objectifs: 1) établir la prévalence des conduites déviantes manifestées au cours de l'adolescence et au début de l'âge adulte par les jeunes femmes ayant été prises en charge par la justice au cours de l'adolescence 2) déterminer les trajectoires de l'activité déviante de ces jeunes femmes, de l'adolescence au début de l'âge adulte 3 ) évaluer la proportion des adolescentes judiciarisées qui sont aux prises avec des difficultés personnelles et sociales au début de l'âge adulte. L'échantillon est composé de 97 jeunes femmes qui ont fait l'objet d'une ordonnance de la Chambre de la jeunesse de Montréal en 1992-1993. Les données proviennent de questionnaires autorévélés qui ont été complétés par les participantes à trois reprises: à 15 ans, à 17 ans et à 23 ans. Les résultats révèlent que, malgré le fait que certaines adolescentes aient été fortement impliquées dans la déviance à la mi-adolescence, aucune trajectoire ne se démarque par une participation persistante à des actes violents ou à des délits contre la propriété. D'autres difficultés compromettent toutefois le bien-être de ces jeunes femmes. Les adolescentes judiciarisées sont particulièrement à risque de se retrouver, au début de l'âge adulte, dans des situations où la monoparentalité, la pauvreté, la faible scolarisation, la violence conjugale et la détresse se juxtaposent.

ABSTRACT - This study has three objectives: 1 ) to establish the prevalence of deviant behaviours reported in adolescence and early adulthood by young women who were adjudicated during their adolescence, 2) to identify the developmental pathways of deviant activities of these young women, 3) to evaluate the proportion of adjudicated adolescent females who are facing personal and social difficulties at the beginning of their adulthood. The sample includes 97 young women who have received a court order from the Youth Court of Montreal in 1992-1993. The data comes from self-reported

1. Le Conseil québécois de la recherche sociale (CQRS), le Conseil de recherches en sciences humaines du Canada (CRSH) et le Fonds pour la formation des chercheurs et l'aide à la recherche (FCAR) ont financé cette recherche.

2. L'auteure désire remercier Marc Le Blanc, professeur à l'École de psychoéducation de l'Université de Montréal, pour l'accès aux données longitudinales. 
questionnaires filled out by participants on three separate occasions: at 15 years old, at 17 years old and at 23 years old. The results show that, even though some young women were strongly involved in deviant activities during their mid-teens, no trajectory denotes a persistent involvement in acts of violence or crimes against property. However, other difficulties compromise the well-being of these young women. Once reaching adulthood, adjudicated adolescent females are particularly at risk of cumulating adversive situations in which single-parenthood, poverty, low levels of education, domestic violence, and distress juxtapose.

La transition entre l'adolescence et l'âge adulte constitue un domaine de recherche très peu documenté en criminologie. En effet, peu d'études ont porté sur les trajectoires qu'empruntent les jeunes filles qui ont fait face à la justice au cours de l'adolescence. Comment évoluent les activités déviantes de ces filles, de l'adolescence à l'âge adulte? Quelle est la proportion de ces filles qui persistent dans la criminalité ou qui restent aux prises avec des problèmes de surconsommation de drogue ou d'alcool? Quel est l'état de leur santé mentale à l'âge adulte? Quel est le cheminement scolaire de ces filles? Se sont-elles intégrées au marché du travail? Une fois l'âge adulte atteint, à quoi ressemble leur situation conjugale et familiale? L'état actuel des connaissances n'offre pas encore de réponses satisfaisantes à ces questions. Cette lacune s'explique par la quasi-absence d'études longitudinales menées auprès de jeunes filles en difficulté et qui couvrent à la fois la période de l'adolescence et celle de l'âge adulte (Lanctôt et Le Blanc, 2002). La récente déclaration de Pajer (1998: 869) en fait foi:

Our understanding of the developmental trajectories of antisocial girls and women is so limited, however, that as policy makers or clinicians, we do not know how to prevent or treat such outcomes.

\section{L'évolution des conduites délinquantes de l'adolescence à l'âge adulte}

Un nombre très restreint d'études abordent l'évolution des conduites délinquantes des filles, de l'adolescence à l'âge adulte. Néanmoins, un constat se dégage des études: les jeunes femmes sont moins nombreuses que leurs pendants masculins à persister dans la délinquance (Lanctôt et Le Blanc, 2002). Tracy et Kempf-Leonard (1996) ont étudié la relation entre la délinquance à l'adolescence et la persistance dans le crime à l'âge adulte. L'analyse des dossiers judiciaires d'une cohorte de 13160 
hommes et de 14000 femmes nés à Philadelphie en 1958 révèle que $12 \%$ des femmes qui ont été arrêtées au cours de leur adolescence l'ont aussi été au début de l'âge adulte. Cette proportion est nettement supérieure chez les hommes, elle se chiffre à $42 \%$. Lewis (1989) rapporte quant à lui que les femmes sont six fois moins nombreuses que les hommes à persister dans le crime. Bien qu'intéressantes, ces études fournissent des résultats incomplets puisque seuls les délits officiellement enregistrés par le système de justice ont été examinés. De plus, seule la prévalence de l'activité délinquante est retenue, au détriment de sa précocité, de sa nature et de sa fréquence.

L'évolution de l'activité délinquante est analysée avec plus de justesse lorsque des sondages de délinquance autorévélée sont mis à profit. Toutefois, la presque totalité des études longitudinales qui privilégient ce type de mesure auprès d'échantillons mixtes ou féminins ne couvre que la période de l'enfance ou de l'adolescence (Dunford et Elliott, 1984 ; Côté et al., 2001 ; Moffitt et al., 2001). C'est donc dire que les études longitudinales qui couvrent à la fois les cycles de vie de l'adolescence et de l'âge adulte ont été réalisées presque uniquement avec des échantillons masculins (Le Blanc et Fréchette, 1989; Laub et Sampson, 2003). L'une des rares études qui met en lumière des trajectoires de l'activité délinquante auprès d'un échantillon mixte est celle de Moffitt et Caspi (2001). Cette étude révèle que dix fois plus d'hommes que de femmes suivent une trajectoire délinquante persistante. L'évolution des conduites délinquantes n'était cependant analysée qu'à partir de deux types de trajectoires: les conduites délinquantes persistantes et les conduites délinquantes limitées à l'adolescence. Cette restriction permet difficilement de rendre compte de l'hétérogénéité des profils comportementaux des adolescentes (Lanctôt et Le Blanc, 2002). Notamment, les adolescentes dont l'activité délinquante commence à un âge plus tardif ne sont pas représentées au sein des trajectoires proposées par Moffitt et Caspi (Silverthorn et Frick, 1999; Silverthorn et al., 2001).

Quelle que soit la nature des mesures utilisées (statistiques officielles ou sondages de délinquance autorévélée), les études recensées concluent que très peu de filles s'engagent avec persistance dans des activités délinquantes. Cette conclusion concerne principalement les activités qui occasionnent un préjudice sérieux à autrui, comme les actes de violence. Devant ce constat, plusieurs chercheurs sont d'avis que les difficultés d'adaptation des jeunes filles délinquantes s'estompent dès 
la fin de l'adolescence (Pajer, 1998). Une telle affirmation mérite toutefois d'être contestée. C'est d'ailleurs ce que fit Robins (1986: 390) en déclarant: "Some of the studies cited above that found that girls did better [than boys] may simply have failed to look at the kinds of outcomes in which antisocial girls do worse».

Cette réplique de Robins (1986) témoigne de la nécessité d'étudier l'évolution d'une variété de conduites inadaptées plutôt que d'analyser uniquement la progression ou le déclin de certains comportements criminels. À cet égard, Broidy et Agnew (1997) notent l'importance d'étudier les comportements, tels que les fugues et la surconsommation de drogues, qui représentent un risque pour la santé et pour la sécurité des jeunes femmes. Selon la recension de Broidy et Agnew (1997), l'importance que les femmes attachent au bien-être d'autrui pourrait expliquer pourquoi elles commettent surtout des actes qui menacent leur propre sécurité plutôt que celle des autres. Enfin, la citation de Robins ne vise pas seulement la nature des comportements qui sont manifestés par les femmes. Elle met aussi en évidence le besoin d'évaluer les trajectoires des jeunes femmes en difficulté en regard du développement de leur adaptation personnelle et sociale.

\section{Le parallèle entre l'évolution des conduites déviantes et l'adaptation personnelle et sociale}

Le cheminement des adolescentes vers l'âge adulte constitue une étape importante de leur développement personnel et social. Bien que l'âge de 18 ans détermine habituellement le passage entre l'adolescence et l'âge adulte, cette référence chronologique n'équivaut pas automatiquement à l'atteinte d'un statut d'adulte qui, lui, s'actualise lorsque les projets de vie (insertion sur le marché du travail, vie de couple, formation d'une famille) se concrétisent. Selon une séquence normative, les jeunes termineraient leur formation scolaire, intégreraient ensuite le marché du travail, emménageraient par la suite avec un(e) conjoint(e) et, finalement, formeraient une nouvelle famille. Or, la réalité se veut nettement plus complexe et désordonnée (Beaupré et Le Bourdais, 2001). Tous les jeunes ne transitent pas dans les mêmes conditions, ni au même rythme, vers un statut adulte. Cette diversité des trajectoires n'est pas sans conséquence puisque certaines avenues sont annonciatrices de difficultés telles que la précarité financière, la monoparentalité, l'isolement social ou les problèmes de santé mentale. 
À cet effet, les jeunes en difficulté d'adaptation, et notamment les filles, sont particulièrement à risque de transiter à un âge précoce vers un statut d'adulte et d'en subir des conséquences négatives (Krohn et al., 1997). L'abandon prématuré du statut d'adolescent peut alimenter les difficultés d'adaptation au fil du temps. Krohn et al. (1997) soulignent qu'un passage précoce vers un statut d'adulte renforce la consommation de drogues au début de la vingtaine. Très peu d'études empiriques ont analysé la relation entre les conduites délinquantes des adolescentes et la qualité de leur adaptation personnelle et sociale à l'âge adulte. La presque totalité des études longitudinales qui s'intéressent à une telle problématique s'effectue exclusivement auprès d'échantillons masculins (Bernburg et Krohn, 2003; Laub et Sampson, 2003; Morizot et Le Blanc, 2003). Quelques études longitudinales qui incluent des filles ont toutefois été recensées.

En ce qui concerne l'adaptation personnelle, des recherches longitudinales indiquent que les troubles de comportement et la délinquance à l'adolescence prédisent des problèmes de santé mentale à l'âge adulte (Wasserman et al., 2003). Cette relation s'observe particulièrement chez les femmes. Les résultats suivants en témoignent. Quinton et al. (1990) rapportent que $35 \%$ des jeunes femmes et $10 \%$ des jeunes hommes qui avaient des troubles de conduite à l'adolescence sont aux prises avec des troubles de l'humeur au début de l'âge adulte. Corneau et Lanctôt (2004) ajoutent que les adolescentes prises en charge par la justice sont plus nombreuses que leurs pendants masculins à rapporter des tentatives de suicide au début de la vingtaine $(21 \%$ et $12 \%$ respectivement). Ces proportions sont très alarmantes si l'on considère que les taux de prévalence des tentatives de suicide se chiffrent autour de $5 \%$ en Amérique du Nord (Institut de la statistique du Québec, 2001).

Par ailleurs, les troubles de comportement et la délinquance à l'adolescence ont également des retombées sur l'adaptation sociale. L'étude de Ayers et ses collègues (1999) et celle de Lanctôt et Le Blanc (2000) indiquent que les adolescentes qui ont des activités délinquantes persistantes accusent d'importants déficits sociaux à la fin de l'adolescence. Ces adolescentes accordent peu d'importance à la réussite scolaire, elles fréquentent bon nombre d'amis délinquants, elles sont peu attachées à leurs parents et elles adhèrent peu aux valeurs prosociales. Ce profil laisse entrevoir un passage difficile vers l'âge adulte. À cet effet, quelques études longitudinales permettent de déterminer les situations adverses auxquelles sont exposées les adolescentes en difficulté, une fois que ces 
dernières atteignent l'âge adulte. Bardone et al., (1996) rapportent que les jeunes filles aux prises avec des troubles de conduite sont particulièrement à risque de se retrouver, au début de l'âge adulte, dans des situations où la monoparentalité, la pauvreté, la faible scolarisation et la violence conjugale se conjuguent. Un profil semblable peut être observé à l'âge adulte pour les jeunes filles qui étaient perçues, selon leurs pairs ou leurs professeurs, comme étant agressives (Serbin et al., 1998) et pour les adolescentes ayant été reconnues coupables d'un délit et mises sous garde (Giordano et al., 2002).

Ces études dressent un profil plutôt sombre de la situation personnelle et sociale des jeunes femmes qui manifestaient des troubles de comportement et des conduites délinquantes au cours de l'adolescence. Elles révèlent un portrait peu reluisant de la situation socioéconomique et familiale de ces jeunes femmes, ainsi que de leur santé mentale. Ce profil n'est pas sans conséquence. Il ne met pas seulement en cause le bien-être des jeunes femmes, il affecte aussi le développement de leurs enfants (Pogarsky, et al., 2003). Le transfert intergénérationnel des difficultés d'adaptation devient alors une préoccupation de taille.

\section{Objectifs}

Les études recensées attestent du peu de connaissances disponibles sur l'évolution de l'activité déviante des adolescentes en difficulté et sur les problèmes d'adaptation auxquels ces dernières sont exposées tout au long de leur cheminement vers l'âge adulte. La présente étude comblera en partie ces lacunes. Trois objectifs sont poursuivis:

1- Établir la prévalence et la nature des conduites déviantes manifestées au cours de l'adolescence et au début de l'âge adulte par les jeunes femmes ayant été prises en charge par la justice au cours de l'adolescence.

2- Déterminer les trajectoires de l'activité déviante des jeunes femmes ayant été prises en charge par la justice au cours de l'adolescence.

3- Évaluer la proportion des adolescentes judiciarisées qui sont aux prises avec des difficultés personnelles et sociales au début de l'âge adulte.

\section{Méthodologie}

Les participantes

L'échantillon est composé d'adolescentes qui ont fait l'objet d'une ordonnance de la Chambre de la jeunesse de Montréal entre les mois 
de février 1992 et de juin 1993. Trois critères de sélection ont permis de constituer l'échantillon. Premièrement, les ordonnances devaient être émises en vertu de la Loi sur la protection de la jeunesse en raison de troubles de comportements sérieux ou en vertu de la Loi sur les jeunes contrevenants. Deuxièmement, ces ordonnances devaient donner lieu à un placement en centre de réadaptation, à un suivi social ou à une période de probation. Enfin, les adolescentes devaient être francophones. Selon ces critères, 172 adolescentes ont été recensées. De ce nombre, seules $22(13 \%)$ n'ont pu être rencontrées, ceci pour des raisons cliniques ou administratives. Il est donc raisonnable d'affirmer que les 150 adolescentes qui composent l'échantillon sont représentatives de l'ensemble des adolescentes francophones qui ont reçu une ordonnance conforme aux critères de sélection.

La majorité des adolescentes $(83 \%, \mathrm{n}=124)$ était sous le couvert de la Loi sur la protection de la jeunesse pour troubles sérieux de comportement. En contrepartie, $17 \%(\mathrm{n}=26)$ des adolescentes de l'échantillon ont été reconnues coupables d'un acte criminel en vertu de la Loi sur les jeunes contrevenants. Le placement en centre de réadaptation fut la mesure la plus fréquemment ordonnée. La presque totalité des adolescentes sélectionnées $(89 \%, \mathrm{n}=109)$ sont nées au Canada. Par contre, elles ne sont que deux tiers $(66 \%, \mathrm{n}=81)$ à déclarer que leur origine ethnique est canadienne. Cet écart s'explique par le fait que le quart des adolescentes $(25 \%, \mathrm{n}=30)$ ont au moins un parent qui a immigré au Canada. D'autre part, très peu d'adolescentes vivent avec leurs deux parents. En effet, pour $83 \%$ d'entre elles $(n=102)$, l'union de leurs parents s'est dissoute. De plus, les adolescentes proviennent majoritairement de familles défavorisées: un peu plus de la moitié des répondantes rapportent que leurs parents $(54 \%, \mathrm{n}=66)$ ont déjà bénéficié de l'aide sociale.

Les adolescentes ont été interviewées à trois reprises, une première fois dans les semaines qui ont suivi l'ordonnance émise en 1992-1993 $(\mathrm{n}=150)$, une seconde fois au cours de l'année 1994-1995 ( $\mathrm{n}=123)$, et une troisième fois en 2000-2001 $(\mathrm{n}=113)$. L'âge moyen était respectivement de 15,2 ans (é.-t. $=1,3$ ), 17,5 ans (é.-t. $=1,4$ ) et 23 ans (é.-t. $=1,5)$ à chaque temps de mesure. Au total, 97 (65\%) adolescentes ont participé aux trois phases de la recherche. Les analyses de la présente étude ne se réferent qu'à ces adolescentes. L'attrition semble avoir été plus élevée parmi les adolescentes qui étaient les moins déviantes au cours de l'adolescence. En effet, les adolescentes qui ont complété les trois phases de la recherche étaient un peu plus impliquées dans la 
consommation de psychotropes et dans les délits contre la propriété comparativement aux adolescentes qui n'ont pas participé à toutes les phases de cette étude longitudinale. Leur implication dans la violence était toutefois similaire.

\section{L'instrument de mesure}

Aux trois temps de mesure, chaque adolescente participait à un entretien structuré à partir d'un questionnaire. Les entretiens duraient environ deux heures et ils se tenaient dans des contextes privés. Ils étaient réalisés à l'aide du Manuel sur des mesures de l'adaptation sociale et personnelle pour les adolescents québécois (MASPAQ [Le Blanc, 1992]). La qualité métrique des variables est plus que satisfaisante, selon les analyses rapportées par Le Blanc (1992). Le MASPAQ permet notamment d'évaluer la position sociale des adolescentes dans la structure sociale, le fonctionnement de leur famille, leurs expériences scolaires et occupationnelles, leurs relations avec leurs amis, leurs activités routinières ainsi que les valeurs auxquelles elles adhèrent. Ce questionnaire évalue aussi l'ampleur et la nature des troubles de comportement et des activités délictueuses manifestées par les participantes. Toutes ces dimensions sont autorévélées.

\section{Les variables et les analyses}

La prévalence des conduites déviantes est rapportée pour la période qui couvre les 12 mois qui ont précédé chaque entrevue. Ces conduites sont liées à des délits contre la propriété ( 9 items), aux conduites violentes (10 items), à la consommation (4 items) et à la vente $(1$ item) de drogues, ainsi qu'à la prostitution (1 item). La variation de la prévalence de chacune de ces conduites dans le temps est estimée à l'aide d'analyses de variance à mesures répétées.

La prévalence des difficultés socioéconomiques et familiales auxquelles sont confrontées les adolescentes judiciarisées, une fois qu'elles atteignent la vingtaine, renvoie quant à elle aux variables suivantes: l'obtention d'un diplôme ou d'une attestation d'études secondaires, l'occupation d'un emploi, la perception de prestations d'aide sociale, la maternité à l'adolescence, la monoparentalité, le suivi des enfants par les services sociaux et la violence entre conjoints. Trois indices témoignent de la santé mentale des jeunes femmes: les tentatives de suicide, 
la consultation d'un professionnel de la santé mentale et la participation à un programme pour contrer la toxicomanie. Toutes ces variables ont été dichotomisées. À titre comparatif, et pour chacun des indicateurs retenus, les prévalences observées au sein de la population générale sont signalées. Ces données comparatives proviennent de diverses enquêtes populationnelles québécoises et canadiennes.

Enfin, les trajectoires de déviance sont définies au moyen d'une analyse par regroupements (cluster analysis). Ce type d'analyse permet de dépister, au sein d'un groupe relativement hétérogène, des cas qui affichent un profil similaire et de les combiner en groupes homogènes. Les paramètres à partir desquels les trajectoires sont définies ont été sélectionnés d'une façon théorique (voir Lanctôt et Le Blanc, 2000). En bref, la précocité et la variété de l'activité déviante sont les paramètres retenus. La précocité détermine l'âge auquel les conduites se sont manifestées pour la première fois, alors que la variété révèle le nombre de conduites différentes au cours des 12 mois précédant chacun des temps de mesure. Ces deux paramètres ont été analysés pour trois échelles de déviance: la violence, les délits contre la propriété ainsi que la consommation de drogues. L'échelle de violence est composée de 10 conduites qui témoignent de la violence physique et des menaces. L'échelle des délits contre la propriété comporte neuf items; elle regroupe les vols mineurs, les vols graves et les actes de vandalisme. Enfin, quatre items se rapportent aux drogues: la consommation de drogues douces, dures et chimiques et la vente de drogues. Les indices de fidélité (alpha de Cronbach) se situent tous au-delà de 0,59.

L'analyse par regroupements s'est effectuée à partir des combinaisons possibles entre les paramètres et les comportements sélectionnés. Douze indicateurs ont été utilisés dans l'analyse par regroupements. Les trois premiers se rapportent à la précocité des activités violentes, à celle des délits contre la propriété et à celle de la consommation de drogues. Les trois indicateurs suivants témoignent de la variété de la violence à chaque temps de mesure, soit approximativement à 15, 17 et 23 ans. Le trio suivant présente la variété des délits contre la propriété commis à chacun des temps de mesure. Enfin, les trois derniers indicateurs attestent de la variété des conduites reliées aux drogues. Puisque les indicateurs ne comportent pas tous le même nombre d'items, les résultats de chaque indicateur ont été standardisés. Ces derniers sont toutefois rapportés dans leur forme brute afin de faciliter leur interprétation. Les valeurs manquantes et les données extrêmes n'entravent pas la rigueur 
des analyses. Une seule participante fut exclue des analyses en raison des valeurs manquantes et quelques valeurs extrêmes ont été recodées à la valeur maximale de leurs échelles respectives. La validité interne des trajectoires fut évaluée à l'aide de divers procédés. Ainsi, la stratégie analytique fut répétée et confirmée avec un sous-échantillon aléatoire. De plus, des analyses de variance attestent que les différences intergroupes sont supérieures aux différences intragroupes.

\section{Résultats}

La prévalence de l'activité déviante rapportée par les jeunes femmes ayant été judiciarisées au cours de leur adolescence est présentée au tableau 1. Pour chaque période à l'étude, ce tableau indique la proportion d'entre elles qui affirment s'être livrées à des activités déviantes telles que la violence, les délits contre la propriété, la consommation de drogues et la prostitution au cours des 12 mois précédant l'entrevue. Pour chaque comportement, le coefficient $\mathrm{F}$ révèle si la prévalence a changé dans le temps.

Se battre à coups de poing, porter une arme et frapper quelqu'un en réaction à une menace ou à une taquinerie sont les activités violentes qui ont été les plus rapportées à chaque période. Toutefois, la proportion des jeunes femmes qui s'adonnent à la violence est relativement faible. Cette constatation se dégage particulièrement à la fin de l'adolescence et au début de l'âge adulte. En effet, à la mi-adolescence, les proportions enregistrées pour les diverses activités violentes variaient entre $43 \%$ et $11 \%$. Les proportions maximales et minimales relevées à la fin de l'adolescence étaient nettement moins élevées (23\% et $3 \%$ respectivement). L'écart est encore plus marqué au début de l'âge adulte, les maximums et minimums observés étant de $16 \%$ et $0 \%$. La proportion des participantes qui se livrent à la violence décline donc considérablement dès la fin de l'adolescence. Les données l'attestent clairement: à la fin de l'adolescence et au début de l'âge adulte, la majorité des conduites violentes ont été rapportées par moins de $10 \%$ des jeunes femmes.

Par ailleurs, les jeunes femmes ayant été judiciarisées au cours de l'adolescence ne se caractérisent pas par leur forte propension à commettre des délits contre la propriété. La prévalence maximale qui fut enregistrée se chiffre à $35 \%$ et elle s'observe à la mi-adolescence pour 
TABLEAU 1

Prévalence de l'activité déviante à trois temps de mesure chez les jeunes femmes ayant été judiciarisées au cours de l'adolescence

\begin{tabular}{|c|c|c|c|c|}
\hline & 15 ans & 17 ans & 23 ans & $\mathbf{F}$ \\
\hline \multicolumn{5}{|l|}{ Violence } \\
\hline Se battre à coups de poing & $43 \%$ & $23 \%$ & $16 \%$ & $11,85 * * *$ \\
\hline Porter une arme & $41 \%$ & $20 \%$ & $13 \%$ & $16,22 * * *$ \\
\hline Frapper en réponse à une menace & $37 \%$ & $14 \%$ & $14 \%$ & $13,30 * * *$ \\
\hline Participer à une bataille de gang & $34 \%$ & $7 \%$ & $3 \%$ & $28,25 * * *$ \\
\hline Encourager les autres à maltraiter qqn & $29 \%$ & $11 \%$ & $8 \%$ & $10,99 * * *$ \\
\hline Utiliser une arme/objet pour se battre & $27 \%$ & $6 \%$ & $0 \%$ & $21,41 * * *$ \\
\hline Menacer pour obtenir quelque chose & $26 \%$ & $11 \%$ & $5 \%$ & $13,21 * * *$ \\
\hline Utiliser la force pour dominer & $15 \%$ & $3 \%$ & $7 \%$ & $5,32 * *$ \\
\hline Menacer pour forcer à faire qqch & $13 \%$ & $4 \%$ & $1 \%$ & $8,01 * *$ \\
\hline Battre quelqu'un qui n'a rien fait & $11 \%$ & $7 \%$ & $0 \%$ & $6,12 * *$ \\
\hline \multicolumn{5}{|l|}{ Délits contre la propriété } \\
\hline Recel & $35 \%$ & $23 \%$ & $12 \%$ & $9,17 * * *$ \\
\hline Vol de moins de $10 \$$ & $35 \%$ & $16 \%$ & $10 \%$ & $13,24 * * *$ \\
\hline Vol à l'étalage & $35 \%$ & $20 \%$ & $7 \%$ & $15,61 * * *$ \\
\hline Introduction par effraction & $33 \%$ & $15 \%$ & $4 \%$ & $17,84 * * *$ \\
\hline Vol entre 10 et $100 \$$ & $30 \%$ & $12 \%$ & $5 \%$ & $14,48 * * *$ \\
\hline Vandalisme & $27 \%$ & $6 \%$ & $5 \%$ & $17,32 * * *$ \\
\hline Vol d'un véhicule moteur & $20 \%$ & $7 \%$ & $1 \%$ & $11,15 * * *$ \\
\hline Vol de plus de $100 \$$ & $19 \%$ & $5 \%$ & $2 \%$ & $10,35 * * *$ \\
\hline Vol par effraction & $15 \%$ & $6 \%$ & $0 \%$ & $10,14 * * *$ \\
\hline \multicolumn{5}{|l|}{ Drogues et prostitution } \\
\hline Consommer des drogues douces & $67 \%$ & $72 \%$ & $78 \%$ & 1,89 \\
\hline Consommer des drogues chimiques & $48 \%$ & $36 \%$ & $46 \%$ & 2,17 \\
\hline Consommer des drogues dures & $45 \%$ & $35 \%$ & $43 \%$ & 1,37 \\
\hline Vendre de la drogue & $32 \%$ & $25 \%$ & $12 \%$ & $7,50 * *$ \\
\hline Se prostituer & $24 \%$ & $11 \%$ & $7 \%$ & $7,99 * * *$ \\
\hline
\end{tabular}

${ }^{*} \mathrm{p} \leq 0,05,{ }^{* *} \mathrm{p} \leq 0,01,{ }^{* * *} \mathrm{p} \leq 0,001$

la possession ou la vente de biens volés, pour les vols de moins de $10 \$$ et pour le vol à l'étalage. Ces délits sont également ceux qui ont été les plus rapportés aux deux temps de mesure subséquents, quoique dans des proportions nettement moindres. Les autres délits contre la propriété dont la gravité relative est supérieure sont peu rapportés et leur 
prévalence décline au fil du temps. Par exemple, dès la fin de l'adolescence, tout au plus $7 \%$ des participantes déclarent avoir soit vandalisé des objets, volé un véhicule moteur, volé pour une somme supérieure à $100 \$$ ou s'être livrées à un vol avec effraction.

Les activités les plus fréquemment rapportées à chacune des périodes sont celles qui ont trait à la consommation de drogues. Les deux tiers des jeunes femmes rapportent avoir consommé des drogues douces au milieu de l'adolescence. Cette proportion passe à trois quarts à la fin de l'adolescence et au début de la vingtaine. La consommation de drogues chimiques et de drogues dures fut rapportée par environ la moitié des adolescentes lors de la première entrevue. Ces proportions diminuent légèrement à la fin de l'adolescence et remontent ensuite au début de l'âge adulte. Les proportions sont alors similaires à celles qui étaient enregistrées à la mi-adolescence. La vente de drogues et la prostitution ont été rapportées par une proportion plus faible de participantes. De plus, ces deux activités déclinent au fil du temps. Alors que le tiers des jeunes femmes déclaraient avoir vendu de la drogue à la mi-adolescence, elles n'étaient plus que $12 \%$ à faire une telle affirmation au début de la vingtaine. De la même façon, $24 \%$ des participantes rapportaient des activités de prostitution au milieu de l'adolescence et seulement $7 \%$ en faisaient autant une fois franchi l'âge adulte.

L'examen de la prévalence des activités déviantes manifestées à l'adolescence et à l'âge adulte suggère que peu de jeunes femmes ayant été judiciarisées au cours de l'adolescence continuent à s'impliquer dans des activités violentes et dans des délits contre la propriété lorsqu'elles franchissent le cap des 18 ans. Les analyses de variance à mesures répétées confirment d'ailleurs le déclin de l'ensemble de ces conduites au fil du temps, mais la consommation de drogues se stabilise. Tel que l'indique l'analyse de variance, la proportion des jeunes femmes qui rapportent consommer de la drogue, qu'elle soit douce, chimique ou dure, demeure stable de l'adolescence au début de l'âge adulte. De façon à mieux évaluer la continuité et le changement de l'activité déviante des adolescentes judiciarisées dans leur cheminement vers l'âge adulte, il est nécessaire d'analyser les variations intra-individuelles de l'activité déviante. En d'autres termes, les conduites déviantes doivent être analysées non pas d'une façon transversale, mais d'un point de vue développemental. 


\section{Les trajectoires}

Les résultats de l'analyse par regroupements mettent en évidence l'hétérogénéité des trajectoires qu'empruntent les adolescentes judiciarisées, du milieu de l'adolescence au début de l'âge adulte. Ces résultats attestent que ces adolescentes ne s'engagent pas toutes au même rythme ni avec la même ampleur dans la déviance. Les analyses dévoilent trois trajectoires caractérisant les adolescentes de cet échantillon: la trajectoire tardive et bénigne $(43 \% ; n=41)$, la trajectoire persistante de consommation de drogues $(31 \% ; \mathrm{n}=30)$ et la trajectoire précoce et déclinante $(26 \% ; n=25)$. Les résultats sont présentés au tableau 2 sous leur forme brute et ils sont illustrés sous une forme standardisée à la figure 1. L'objectif recherché est atteint, c'est-à-dire optimiser l'homogénéité

TA B LEA U 2

Analyse de variance et résultats moyens des trois trajectoires

\begin{tabular}{|c|c|c|c|c|}
\hline & & $\begin{array}{c}\text { Tardive } \\
\text { et bénigne } \\
\mathrm{N}=41\end{array}$ & $\begin{array}{c}\text { Consommation } \\
\text { persistante } \\
\text { de drogues } \\
\mathrm{N}=31\end{array}$ & $\begin{array}{l}\text { Précoce et } \\
\text { déclinante } \\
\mathrm{N}=25\end{array}$ \\
\hline & $\mathbf{F}$ & M (é.-t.) & M (é.-t.) & M (é.-t.) \\
\hline \multicolumn{5}{|l|}{ Précocité } \\
\hline Violence & $21,91 * * *$ & $13,76(3,60)$ & $11,57(2,96)$ & $8,48(2,49)$ \\
\hline Délits contre propriété & $15,38 * * *$ & $14,39(4,82)$ & $10,97(2,74)$ & $9,52(2,28)$ \\
\hline Drogues & $30,23 * * *$ & $15,93(2,93)$ & $12,60(1,47)$ & $11,80(2,04)$ \\
\hline \multicolumn{5}{|l|}{$\begin{array}{l}\text { Variété }- \text { violence } \\
(\min .=0 ; \max .=10)\end{array}$} \\
\hline Mi-adolescence & $42,71 * * *$ & $1,39(2,14)$ & $2,03(1,88)$ & $5,96(1,97)$ \\
\hline Fin de l'adolescence & 3,08 * & $0,56(1,22)$ & $1,37(1,97)$ & $1,40(1,66)$ \\
\hline Début de la vingtaine & $2,52 \dagger$ & $0,46(1,16)$ & $0,56(1,07)$ & $1,16(1,60)$ \\
\hline \multicolumn{5}{|l|}{$\begin{array}{l}\text { Variété }- \text { propriété } \\
(\min .=0 ; \max .=9)\end{array}$} \\
\hline Mi-adolescence & $42,49 * * *$ & $1,00(1,55)$ & $2,03(1,99)$ & $5,60(2,59)$ \\
\hline Fin de l'adolescence & $7,27 * * *$ & $0,37(1,07)$ & $1,57(2,01)$ & $1,84(2,11)$ \\
\hline Début de la vingtaine & 5,05 * & $0,17(0,38)$ & $0,87(1,38)$ & $0,44(0,82)$ \\
\hline \multicolumn{5}{|l|}{$\begin{array}{l}\text { Variété }- \text { drogue } \\
(\min .=0 ; \max .=4)\end{array}$} \\
\hline Mi-adolescence & $49,94 * * *$ & $0,61(0,89)$ & $2,80(1,03)$ & $2,92(1,41)$ \\
\hline Fin de l'adolescence & $21,09 * * *$ & $0,80(1,00)$ & $2,70(1,18)$ & $1,80(1,55)$ \\
\hline Début de la vingtaine & $21,26 * * *$ & $1,17(0,89)$ & $2,70(0,75)$ & $1,64(1,32)$ \\
\hline
\end{tabular}

$\dagger p \leq 0,10,{ }^{*} p \leq 0,05, * * p \leq 0,01,{ }^{* * *} p \leq 0,001$ 
FIGURE 1

Les trajectoires déviantes des adolescentes judiciarisées de l'adolescence à l'âge adulte

Précocité

intragroupe tout en maximisant les différences qui distinguent les groupes. En effet, l'analyse de variance rapportée au tableau 2 indique que, pour chaque indicateur, la variance intragroupe est inférieure à la variance intergroupe.

Selon les profils moyens des adolescentes qui appartiennent à chacune des trajectoires, celles qui affichent un profil tardif et bénin $(n=41)$ sont celles qui se sont initiées le plus tardivement aux activités déviantes. Elles ont manifesté leurs premiers comportements violents vers 14 ans et commis leurs premiers délits contre la propriété vers 14 ans et demi. Quant à la consommation des psychotropes, elle fut initiée tout juste avant 16 ans. Ces adolescentes se distinguent également par la faible diversification de leurs activités déviantes. En effet, la violence, les délits contre la propriété et la consommation des psychotropes se sont manifestés sous des formes peu variées comparativement au répertoire des activités déviantes des adolescentes des autres trajectoires. Cette faible variété est apparente au milieu de l'adolescence, à la fin de l'adolescence et au début de la vingtaine. Leur faible implication dans la délinquance se traduit par un résultat maximum de 1,39 (sur une échelle pouvant atteindre 10) pour la violence et par un résultat maximum de 1,00 (sur une échelle pouvant atteindre 9) pour les délits contre la propriété. Un profil similaire se dégage de leur consommation de drogues. Sur une possibilité de quatre conduites, elles en ont rapporté en moyenne tout au plus 1,17 . 
À l'autre extrême se situent les adolescentes de la trajectoire précoce et déclinante $(\mathrm{n}=25)$. Ces adolescentes ont fait une entrée précoce sur la scène de la déviance, soit environ cinq années plus tôt que leurs consœurs qui suivent une trajectoire tardive et bénigne. En effet, dès l'âge de 8 ans et demi, des actes de violence se sont manifestés. L'initiation aux délits contre la propriété a suivi environ un an plus tard, à 9 ans et demi. La consommation des psychotropes a également débuté à un âge précoce, soit tout juste avant 12 ans. Les adolescentes de cette trajectoire se démarquent également par la variété de leurs activités déviantes, particulièrement au milieu de l'adolescence. Au cours de cette période, elles rapportent s'être livrées, en moyenne, à près de six des dix conduites violentes et à 5,6 des neuf délits contre la propriété. Elles déclaraient aussi avoir participé à trois $(2,92)$ des quatre conduites relatives aux drogues. C'est donc dire qu'à la mi-adolescence, leurs activités délinquantes et leur consommation de drogues étaient entre quatre et six fois plus variées que celles des adolescentes de la trajectoire tardive et bénigne. Toutefois, dès la fin de l'adolescence, leur délinquance décline. Leurs activités délinquantes, qu'il s'agisse des délits violents ou de ceux contre la propriété, se résorbent considérablement jusqu'au début de la vingtaine. Leur consommation de drogues diminue quant à elle plus lentement.

Entre ces deux trajectoires opposées se trouve la trajectoire qui dénote une consommation persistante de drogues $(\mathrm{n}=30)$. Globalement, la précocité des activités déviantes des jeunes femmes de cette trajectoire affiche des résultats moyens comparativement aux deux autres trajectoires. Ces jeunes femmes se caractérisent toutefois par la stabilité de leur consommation de drogues. En effet, de la mi-adolescence au début de la vingtaine, elles rapportent en moyenne près de trois des quatre conduites reliées aux drogues. Leur activité délinquante, qu'elle soit de nature violente ou contre la propriété, semble quant à elle n'être qu'exploratoire. À la mi-adolescence, ces jeunes femmes rapportaient près de trois fois moins d'actes violents et de délits contre la propriété que les jeunes femmes de la trajectoire la plus délinquante. De plus, ces activités délinquantes étaient de moins en moins rapportées avec le temps.

En bref, l'établissement des trajectoires révèle qu'aucun sous-groupe d'adolescentes judiciarisées ne se démarque par une participation persistante à des actes violents ou à des délits contre la propriété, ceci malgré le fait que certaines adolescentes aient été fortement impliquées dans la délinquance à la mi-adolescence. Un sous-groupe, qui compose environ 
le tiers de l'échantillon, consomme toutefois des drogues avec persistance. La question à se poser maintenant est la suivante: les difficultés d'adaptation auxquelles sont exposées les adolescentes judiciarisées au moment de la transition vers l'âge adulte se reflètent-elles davantage dans leur statut économique et familial, et leur santé mentale?

\section{Le profil social et personnel}

Les résultats du tableau 3 indiquent que les jeunes femmes qui ont été sous le couvert de la justice au cours de l'adolescence se retrouvent, à l'âge adulte, dans une situation sociale et personnelle précaires. Ces proportions sont calculées en fonction de ce que les jeunes femmes rapportaient au moment de l'entrevue réalisée alors qu'elles avaient en moyenne 23 ans. À titre comparatif, les prévalences observées au sein de la population générale sont signalées.

D'abord, plus des deux tiers (68\%) des jeunes femmes ayant fait face à la justice au cours de l'adolescence affirment ne pas avoir obtenu de diplôme ou d'attestation d'études secondaires. Une proportion semblable (69\%) signale être sans emploi. Au moment de l'entrevue, plus de la moitié $(56 \%)$ des jeunes femmes ont affirmé recevoir des prestations d'aide sociale. Cette condition socioéconomique précaire s'explique peut-être par le fait que ces jeunes femmes sont nombreuses (44\%) à avoir eu leur premier enfant avant 20 ans, ce qui rendait difficile la poursuite de leurs études. De plus, la plupart $(61 \%)$ des jeunes mères assument seules la garde de leur(s) enfant(s). Ces difficultés auxquelles se heurtent les jeunes mères ne sont pas sans conséquence. Les services sociaux, notamment, interviennent auprès des enfants de $23 \%$ des jeunes mères, selon les informations dévoilées par ces dernières. Plus encore, lorsqu'elles vivent en couple, la moitié des jeunes femmes affirment avoir subi (52\%) ou avoir manifesté $(50 \%)$ de la violence physique entre conjoints.

À ce sombre profil social se greffe une vulnérabilité personnelle. Une fois l'âge adulte atteint (18 ans), environ le quart (23\%) des adolescentes judiciarisées révèle avoir tenté de se suicider. Le tiers (32\%) rapporte avoir consulté un psychologue depuis leur $18^{\mathrm{e}}$ anniversaire. Enfin, $15 \%$ des jeunes femmes affirment avoir participé à un programme ou à une cure de désintoxication au cours de cette même période. Ce profil social et personnel accuse de lourds déficits si on le compare à celui des jeunes femmes de la population générale. Pour chacun des 
TA B LEA U 3

Prévalence des difficultés sociales et personnelles des jeunes femmes ayant été judiciarisées à l'adolescence

\begin{tabular}{|lrcc|}
\hline & & \multicolumn{2}{c|}{$\begin{array}{c}\text { Prévalence au début } \\
\text { de la vingtaine }\end{array}$} \\
\cline { 3 - 4 } & & $\begin{array}{c}\text { Adolescentes } \\
\text { judiciarisées }\end{array}$ & $\begin{array}{c}\text { Population } \\
\text { générale }\end{array}$ \\
\hline Ne pas avoir de diplôme d'études secondaires & $(n=97)$ & $68 \%$ & $15 \%^{3}$ \\
Ne pas occuper un emploi & $(n=92)$ & $69 \%$ & $12 \%^{4}$ \\
Bénéficier de l'aide sociale & $(n=92)$ & $56 \%$ & $11 \%^{5}$ \\
Avoir eu son 1' $1^{\text {er }}$ enfant avant 20 ans & $(n=97)$ & $44 \%$ & $14 \%^{6}$ \\
Être mère monoparentale & $(n=60)$ & $61 \%$ & $21 \%^{7}$ \\
Avoir un enfant suivi par les services sociaux & $(n=58)$ & $23 \%$ & $6 \%{ }^{8}$ \\
Avoir été violentée par son conjoint & $(n=52)$ & $52 \%$ & $14 \%{ }^{9}$ \\
Avoir violenté son conjoint & $(n=52)$ & $50 \%$ & $13 \%{ }^{10}$ \\
Avoir tenté de se suicider & $(n=97)$ & $23 \%$ & $6 \%{ }^{11}$ \\
Avoir consulté un spécialiste de la santé mentale & $(n=97)$ & $32 \%$ & $13 \% 0^{12}$ \\
Avoir suivi un programme de désintoxication & $(n=97)$ & $15 \%$ & $<1 \%{ }^{13}$ \\
\hline
\end{tabular}

indicateurs, les jeunes femmes qui ont fait face à la justice au cours de l'adolescence affichent des taux de prévalence qui dépassent d'au moins trois fois, si ce n'est pas plus, ceux de leurs consœurs représentatives de la population québécoise.

3. Institut de la statistique du Québec, 2001-2002, populative féminine québécoise de $15-24$ ans.

4. Institut de la statistique du Québec, 2001-2002, populative féminine québécoise de $15-24$ ans.

5. Institut de la statistique du Québec, 2001-2002, populative féminine québécoise de $15-24$ ans.

6. Institut de la statistique du Québec, 2001-2002, populative féminine québécoise de 15-24 ans.

7. Statistique Canada, 2001-2002, populative féminine québécoise de 15 ans et plus.

8. Ministère de la Santé et des Services sociaux du Québec et Institut de la statistique du Québec, 2001, population québécoise.

9. Laporte et Chamberland, 2002, échantillon populationnel montréalais, jeunes femmes de 12 à 24 ans.

10. Laporte et Chamberland, 2002, échantillon populationnel montréalais, jeunes femmes de 12 à 24 ans.

11. Santé Québec, 1996, prévalence des idéations suicidaires au cours de la vie, population féminine québécoise 15-24 ans

12. Statistique Canada, 2001-2002, population féminine canadienne de 15 ans-24 ans

13. Direction de la santé publique de Montréal, 1993, population montréalaise de 15 ans et plus. 
Par ailleurs, des analyses bivariées (non présentées ici) rapportent que ce profil à l'âge adulte se ressemble, peu importe la trajectoire empruntée par les jeunes femmes. Seule la participation à un programme de désintoxication distinguait les trajectoires à l'âge adulte, les jeunes femmes consommatrices de drogues y étant surreprésentées. Cette quasiabsence de distinctions entre les femmes de chaque trajectoire, quant à la qualité de leur adaptation à l'âge adulte, a de quoi étonner: les jeunes femmes qui ont une consommation persistante de drogues ne semblent pas confrontées à plus d'adversité au début de la vingtaine comparativement à celles qui suivent une trajectoire de déviance bénigne ou déclinante. Une analyse plus approfondie sera de mise, notamment en considérant le cumul des difficultés socioéconomiques, sociales et personnelles à l'âge adulte plutôt que la prévalence de chaque indicateur pris isolément.

\section{Discussion}

Cette étude avait trois objectifs: 1) établir la prévalence des conduites déviantes manifestées au cours de l'adolescence et au début de l'âge adulte par les jeunes femmes ayant été prises en charge par la justice au cours de l'adolescence 2) déterminer les trajectoires de l'activité déviante des jeunes femmes ayant été prises en charge par la justice au cours de l'adolescence 3) évaluer la proportion des adolescentes judiciarisées qui sont aux prises avec des difficultés personnelles et sociales au début de l'âge adulte.

Les résultats montrent que la plupart des adolescentes judiciarisées délaissent les activités délinquantes lorsqu'elles transitent vers l'âge adulte. En effet, la participation de ces jeunes femmes à des actes violents ou à des délits contre la propriété est rare dès la fin de l'adolescence. Ces résultats correspondent aux quelques écrits qui portent sur l'évolution des conduites déviantes des adolescentes. Ainsi, la détermination des trois trajectoires confirme que la persistance dans la délinquance caractérise peu les adolescentes (Lewis, 1989; Tracy et Kempf-Leonard, 1996; Moffitt et al., 2001). Ce profil comportemental est somme toute positif. Malgré le fait que plusieurs jeunes femmes s'adonnaient à la délinquance au milieu de l'adolescence, très peu empruntent la voie de la criminalité à l'âge adulte.

Par contre, derrière cette apparence de conformité se cachent des difficultés marquées. D’une part, la consommation de drogues demeure 
très présente pour environ le tiers des jeunes femmes de l'échantillon. Plus important encore, les jeunes femmes qui ont été confrontées à la justice au cours de leur adolescence se retrouvent, pour la majorité, dans une position sociale précaire au début de la vingtaine. La majorité d'entre elles n'ont pas complété leurs études secondaires, plus des deux tiers n'occupent pas un emploi et plus de la moitié dépendent financièrement des prestations d'aide sociale. À ces difficultés socioéconomiques se conjugue une situation familiale qui, bien souvent, est marquée par la monoparentalité et par la violence. Un profil similaire était rapporté auprès d'échantillons de la Nouvelle-Zélande (Bardone et al., 1996) et des États-Unis (Giordano et al., 2002). La santé mentale de ces jeunes femmes se caractérise aussi par la fragilité, surtout lorsqu'elle est comparée à celle des jeunes femmes de la population générale. Ces résultats rejoignent les préoccupations de Robins (1986) et celles de Broidy et Agnew (1997). Plutôt que de se livrer à des gestes qui causent de sérieux préjudices à autrui, les jeunes femmes en difficulté tendent à se retrouver dans des situations qui compromettent leur propre développement et leur sécurité.

Plus encore, les analyses bivariées, bien qu'exploratoires, suggèrent que les adolescentes judiciarisées risquent fortement de se retrouver dans une situation socioéconomique et personnelle très précaire au début de l'âge adulte, cela indépendamment de la trajectoire de déviance qu'elles empruntent. Bien que des analyses plus approfondies seront nécessaires afin d'évaluer les conséquences de la délinquance d'une façon plus complète et intégrative, certaines explications peuvent être avancées. La théorie développementale de Sampson et Laub (1997), qui réfere au cumul des désavantages au cours de la vie, aide à comprendre comment le passage dans le système de justice peut compromettre le développement social et personnel des individus à travers les cycles de la vie:

The theory specifically suggests a «snowball» effect that adolescent delinquency and its negative consequences (e.g., arrest, official labeling, incarceration) increasingly "mortage» one's future, especially later life chances molded by schooling and employment. (Sampson et Laub, $1997: 47$ )

Deux perspectives théoriques sont intégrées à ce modèle : celle de la régulation sociale de la déviance et celle de l'étiquetage des personnes judiciarisées. D'une part, la transgression des normes sociales à l'adolescence diminue la force des liens familiaux et scolaires en plus de favoriser l'intériorisation de valeurs antisociales et la fréquentation de pairs 
délinquants. Ces conditions ne sont pas favorables à une transition réussie vers un statut d'adulte. Cette explication s'applique à nos résultats puisque les adolescentes qui composaient notre échantillon avaient toutes été judiciarisées en raison de leurs troubles sérieux de comportement ou en raison d'un délit qu'elles avaient commis. D'autre part, Sampson et Laub (1997) ajoutent que des conséquences négatives peuvent résulter d'un passage dans le système de justice. C'est le cas de la stigmatisation qui accompagne une prise en charge par la justice; elle peut nuire à l'établissement de liens sociaux avec les institutions conventionnelles, et ce, indépendamment des prédispositions comportementales des individus. Encore une fois, cette perspective s'applique à nos résultats puisque ces derniers portent sur des adolescentes ayant été judiciarisées.

En somme, les résultats suggèrent que les adolescentes judiciarisées représentent une priorité en matière d'intervention sociale. Bon nombre de ces jeunes femmes sont aux prises avec des difficultés sociales et personnelles et plusieurs assument seules la garde de leurs enfants. Cependant, l'analyse ne permet pas d'évaluer si le profil de ces jeunes femmes se distingue de celui des jeunes hommes ayant aussi été judiciarisés au cours de leur adolescence. Une telle analyse devra ultérieurement être effectuée afin d'établir la nécessité d'élaborer des stratégies d'intervention qui soient particulières ou non à la clientèle féminine. Enfin, l'évaluation des composantes de l'adaptation personnelle et sociale qui favorisent une saine transition vers l'âge adulte dépassait les objectifs de cet article. Cette évaluation devra assurément être effectuée afin de mieux déterminer les jeunes femmes les plus à risque et de mieux définir les programmes d'intervention destinés aux jeunes filles en difficulté.

Quelques implications pratiques se dégagent de la présente étude. Les résultats attestent sans contredit que les adolescentes judiciarisées cumulent leurs difficultés au fil du temps. Bien qu'une proportion des jeunes femmes puisse échapper à l'adversité, il reste que la majorité est confrontée à des obstacles qui restreignent grandement leur bien-être. À ce titre, la faible scolarisation des adolescentes judiciarisées constitue une entrave importante à leur intégration sociale. Il va de soi que des programmes doivent être offerts aux adolescentes en difficulté afin de favoriser leur insertion sur le marché du travail. Le programme «Ma place au soleil» est un modèle à retenir. Ce programme est offert par le ministère de la Sécurité du revenu et par Emploi-Québec (Sécurité du revenu, Québec, 
2003). Il repose sur une démarche d'intégration en emploi par l'entremise d'une formation diplômée qui peut durer jusqu'à 36 mois. Cette démarche vise l'obtention d'un diplôme d'études professionnelles, d'une attestation de spécialisation professionnelle ou d'une attestation d'études collégiales. Ce programme s'adresse aux jeunes mères prestataires de l'assistance-emploi qui désirent effectuer un retour aux études tout en recevant du soutien pour leurs responsabilités familiales. La priorité est accordée aux responsables de famille monoparentale. Les prémisses d'un tel programme reposent sur le respect des participantes et sur la souplesse de l'encadrement. En outre, au cours de leur participation au programme, les jeunes mères reçoivent, en plus de leur prestation d'assistance-emploi, le remboursement des frais de garde et de transport. Ce programme prévoit aussi des activités de groupe qui permettent aux jeunes mères de créer des liens entre elles et de s'entraider au quotidien. Ces initiatives peuvent contribuer à amoindrir l'isolement social et la détresse qui, trop souvent, l'accompagne. Ce programme correspond très bien aux besoins des adolescentes judiciarisées, tel qu'en témoigne le profil de leur adaptation personnelle et sociale.

Offrir des moyens aux jeunes femmes pour qu'elles s'émancipent et pour qu'elles rompent avec les contraintes associées à la dépendance économique et la monoparentalité est un objectif louable. Un tel objectif pourrait toutefois difficilement se réaliser si les besoins cognitifs, émotifs et affectifs de ces jeunes femmes n'étaient pas reconnus. En effet, il faut admettre que, outre la précarité de leur situation socioéconomique, les jeunes femmes judiciarisées au cours de leur adolescence doivent acquérir des moyens afin d'exprimer convenablement leur colère et de régler leurs problèmes d'une façon saine. La forte prévalence de la violence entre conjoints rapportée dans la présente étude en témoigne. Elles doivent aussi apprendre à mieux composer avec les tensions qui découlent des événements difficiles auxquelles elles sont exposées, les problèmes de toxicomanie et les tentatives de suicide que nous avons évoqués en font foi. Plus important encore, ces adolescentes doivent être mieux outillées pour pouvoir considérer et appliquer des solutions de rechange lorsqu'elles sont confrontées à des situations difficiles. Ces composantes font actuellement partie intégrante des stratégies de réadaptation destinées aux adolescentes qui bénéficient d'un placement au Centre jeunesse de Montréal. Il reste maintenant à vérifier si un tel programme, ou un autre tel celui décrit plus haut, améliorera le devenir des adolescentes en difficulté. 


\section{Références}

Ayers, C.D., Williams, H., Hawkins, J.D., Peterson, P.L., Catalano, R.F., \& Abbott. R.F. (1999). Assessing correlates of onset, escalation, and desistance of delinquent behavior. Journal of Quantitative Criminology, 15, 277-305.

Bardone, A.M., Moffitt, T.E., Caspi, A., Dickson N., \& Silva. P.A. (1996). Adult mental health and social outcomes of adolescent girls with depression and conduct disorder. Development and Psychopathology, 8, 811-829.

Beaupré, P., \& Le Bourdais, C. (2001). Le départ des enfants du foyer parental au Canada. Cabiers québécois de démographie, 30 (1), 29-62.

Bernburg,. J.G., \& Krohn, M.D. (2003). Labeling, life chances, and adult crime: the direct and indirect effects of official intervention in adolescence on crime in early adulthood. Criminology, 41 (4), 1287-1318.

Broidy, L., \& Agnew, R. (1997). Gender and crime: a general strain theory perspective. Journal of Research in Crime and Delinquency, 34 (3), 275-306.

Corneau, M., \& Lanctôt N. (sous presse). Mental health outcomes of adjucicated youths: the aftermath of juvenile delinquency. Criminal Behaviour and Mental Health.

Côté, S., Zoccolillo, M., Tremblay, R.E., \& Nagin, D. (2001). Predicting girls' conduct disorder in adolescence from childhood trajectories of disruptive behaviors. Journal of the American Academy of Child and Adolescent Psychiatry, 40, 678-84.

Direction de la santé publique de Montréal-Centre. Rapport annuel 1999 sur la santé de la population. Prévenir, guérir, soigner. Les défis d'une société vieillissante. Décembre 1999.

Dunford, F.W., \& Elliott, D.S. (1984). Identifying career offenders using selfreported data. Journal of Research in Crime and Delinquency, 21 (1), 57-86.

Giordano P.C., Cernkovich, S.A., \& Rudolph, J.L. (2002). Gender, crime, and desistance: toward a theory of cognitive transformation. American Journal of Sociology, 107 (4), 990-1064.

Institut de la statistique du Québec (2002). Rapport annuel 2001-2001. Québec.

Krohn, M.D., Lizotte, A.J., \& Perez, C.M. (1997). The interrelationships between substance use and precocious transitions to adult statuses. Journal of Health and Social Behavior, 38 (1), 87-103.

Lanctôt, N., \& Le Blanc M. (2000). Les trajectoires marginales chez les adolescentes judiciarisées: continuité et changement. Revue Internationale de Criminologie et de Police Technique et Scientifique, 53 (1), 46-68.

Lanctôt, N., \& Le Blanc, M. (2002). Explaining adolescent females' involvement in deviance. Crime and Justice, 29, 113-202.

Laporte, L., \& Chamberland, C. (2002). La violence dans les relations amoureuses: qu'en pensent nos jeunes? Défi jeunesse, 8 (2), 13-20.

Laub, J.H., \& Sampson, R.J. (2003). Shared beginning, divergent lives : delinquent boys to age 70. Cambridge: Havard University Press.

Le Blanc, M. (1992). MASPAQ Manuel sur des mesures de l'adaptation sociale et personnelle pour les adolescents québécois. Montréal: Groupe de recherche sur l'inadaptation psycho-sociale à l'enfance, Université de Montréal. 
Le Blanc, M., \& Fréchette, M. (1989). Male criminal activity from childhood to adulthood: multilevel and developmental perspectives. New York: Springer-Verlag.

Lewis, R.V. (1989). Does there exist an adult female chronic offender? Communication présentée au $41^{\mathrm{e}}$ Congrès de l'American Society of Criminology, Reno, LV, novembre.

Ministère de la Santé et des Services Sociaux du Québec (2002-2003). Rapport annuel de gestion. Québec.

Moffitt, T.E., \& Caspi, A. (2001). Childhood predictors differentiate life-course persistent and adolescence-limited antisocial pathways among males and females. Development and Psychopathology, 13, 355-375.

Moffitt, T.E., Caspi, A., Rutter, M., \& Silva, P.A. (2001). Sex differences in the effects of antisocial behaviour on young adults outcomes. In T.E. Moffitt, A. Caspi, M. Rutter \& P.A. Silva (eds), Sex differences in antisocial behaviour, conduct disorder, delinquency, and violence in the Dunedin Longitudinal Study (159183). U.K. : Cambridge University Press.

Morizot, J. \& Le Blanc, M. (2003). Searching for a developmental typology of personality and its relations to antisocial behaviour: a longitudinal study of adjudicated men sample. Criminal Behaviour and Mental Health, 13, 241-277.

Pajer, K.A. (1998). What happens to bad girls? A Review of adult outcomes of antisocial adolescent girls. American Journal of Psychiatry, 155, 862-870.

Pogarsky, G., Lizotte, A.J., \& Thornberry, T.P. (2003). The delinquency of children born to young mothers: results from the Rochester youth development study. Criminology, 41 (4), 1249-1286.

Quinton, D., Rutter, M., \& Gulliver, L. (1990). Continuities in psychiatric disorders from childhood to adulthood in children of psychiatric patients. In L.N. Robins \& M. Rutter (eds), Straight and devious pathways from childhood to adulthood (259-278). Cambridge: Cambridge University Press.

Robins, L.N. (1986). The consequences of conduct disorder in girls. In D.C. Farran \& J.C. McKinney (eds), Risk in Intellectual and psychosocial development. Orlando: Academic Press.

Sampson, R.J., \& Laub, J.H. (1997). A life-course theory of cumulative disadvantage and the stability of delinquency. In T.P. Thornberry (ed.), Developmental theories of crime and delinquency, vol. 7, Advances in criminological theory. New Brunswick (USA): Transaction Publishers.

Santé Québec (1996). Enquête sociale et de santé 1992-1993. Montréal: Ministère de la Santé et des Services sociaux, gouvernement du Québec.

Sécurité du revenu, Québec (2003). Ma place au soleil. Site internet de la Sécurité du revenu, Québec, consulté le 10 août 2004, <http://www.mess. gouv.qc.ca/francais/jeunesse/maplaceausoleil $>$.

Serbin, L.A., Cooperman, J.M., Peters, P.L., Lehoux, P., Stack, D.M., \& Schwartzman, A.E. (1998). Intergenerational transfer of psycho social risk in women with childhood histories of aggression, withdrawal or aggression and withdrawal. Developmental Psychology, 34, 1246-1262.

Silverthorn, P., \& Frick, P.J. (1999). Developmental pathways to antisocial behavior: the delayed-onset pathway in girls. Development and Psychopathology, 11, 101-126. 
Silverthorn, P., Frick, P.J., \& Reynolds, R. (2001). Timing of onset and correlates of severe conduct problems in adjudicated girls and boys. Journal of Psychopathology and Behavioral Assessment, 23 (3),171-181.

Statistique Canada (2002). Enquête sur la santé dans les collectivités canadiennes. Santé mentale et bien-être. Canada.

Tracy, P.E., \& Kempf-Leonard, K. (1996). Continuity and discontinuity in criminal careers. New York: Plenum Press.

Wasserman, G.A., McReynolds, L.S., \& Ko, S.J. (2003). Gender differences in psychiatric disorder among juvenile justice youths. Center for the promotion of mental health in juvenile justice, Columbia University, Division of Child Psychiatry. Document inédit. 